\title{
IP Mobility Support with a Multihomed Mobile Router
}

\author{
Hee-Dong Park ${ }^{1}$, Dong-Won Kum², Yong-Ha Kwon ${ }^{2}$, \\ Kang-Won $\mathrm{Lee}^{2}$, and You-Ze $\mathrm{Cho}^{2}$ \\ ${ }^{1}$ Department of Computer Engineering, Pohang College, Pohang, 791-711, Korea \\ hdpark@pohang. ac . kr \\ ${ }^{2}$ School of Electrical Engineering \& Computer Science, \\ Kyungpook National University, Daegu, 702-701, Korea
}

\begin{abstract}
This paper proposes a multihoming-based seamless handover scheme using a mobile router with dual egress interfaces for wireless train networks. The proposed scheme deploys dual antennas which are individually located at each end of the train for space diversity and connected to each egress interface of a mobile router. Since one of the two egress interfaces of the mobile router can continuously receive packets through its antenna while the other is undergoing a handover, the proposed scheme can support a seamless handover providing no service disruption or packet loss.
\end{abstract}

\section{Introduction}

Network mobility (NEMO) basic support is concerned with managing the mobility of an entire network [1]. Public transportation, such as trains and buses, is an example of the mobile networks [2]. The NEMO basic protocol will be built on Mobile IPv6 with minimal extensions [3]. Therefore, the handover mechanism of a mobile router (MR) is essentially the same as that of a mobile node (MN) with Mobile IP. Recently, various multihoming issues have been presented in the NEMO Working Group. The multihoming is necessary to provide constant access to the Internet and to enhance the overall connectivity of hosts and mobile networks [4][5]. This requires the use of several interfaces and technologies since the mobile network may be moving in distant geographical locations where different access technologies are provided. The additional benefits of the multihoming are fault tolerance/redundancy, load sharing, and policy routing. However, there is no requirement or protocol defining how to use several interfaces with a mobile network. This paper proposes a multihoming-based handover scheme using an MR with dual egress interfaces, which cooperate with each other to perform seamless handovers for a large moving network, such as trains. The proposed scheme deploys dual antennas which are individually located at each end of the moving network for space diversity and connected to each egress interface of the MR. One of the two egress interfaces can continuously receive packets through its antenna, while the other is undergoing a handover. This can support a seamless handover providing no service disruption or packet loss. 


\section{Multihoming-Based Seamless Handover}

The proposed system is assumed to be deployed in a large mobile network such as a train. Fig. 1 shows the vehicle network structure of the proposed scheme. For multihoming, an MR with dual antennas (Head_ANT and Tail_ANT) can be deployed in the vehicle. The Head_ANT and Tail_ANT are located in the front and back end of the train, respectively. The multihomed MR has at least two egress interfaces connected to the dual antennas, and each of the two interfaces has its own HoA and CoA. In the proposed scheme, the terms Head_CoA and Head_HoA are used to represent the CoA and HoA of the interface connected to the Head_ANT, while the terms Tail_CoA and Tail_HoA are used for the interface connected to the Tail_ANT. Also, Mobile IPv6 is assumed to be used for the proposed system. There are APs in each car of the train, and they are connected to the MR through a switch.

Fig. 2 shows the handover procedure of the proposed scheme. When both antennas stay in the Old_AR's coverage area, the MR communicates with the Old_AR through the Tail_ANT, while the Head_ANT waits for an impending handover.

(1) Phase 1: As the mobile network moves, the Head_ANT reaches New_AR's coverage area prior to the Tail_ANT. After the MR receives the network prefix information from the New_AR through the Head_ANT and associates with the New_AR by creating a CoA (Head_CoA), it sends a proxy BU message to the HA. The proxy BU message contains the new Head_CoA and the Tail_HoA instead of the Head_HoA. This makes the HA to be under the illusion that the MR has only one egress interface, and prevents the HA from having multiple bindings. The Tail_ANT, however, actually continues to receive packets in the Old_ARs coverage area, thus packet loss can be prevented. After receiving the Proxy BU message, HA updates the binding and delivers packets to the MR through the New_AR. When the MR receives a Proxy BU ACK message from the HA through the Head_ANT, it sends the data packets originated from the MNNs to the Internet through the Head_ANT.

(2) Phase 2: When the Tail_ANT stays in the Old_AR's coverage area and the Head_ANT stays in the New_AR's coverage area simultaneously, the MR can send and receive data packets through the Head_ANT, and it may also receive in-transit data packets destined to the Old_AR through the Tail_ANT.

(3) Phase 3: If the MR receives router advertisement messages from the New_AR through the Tail_ANT, the MR performs a handover. At this time, the MR

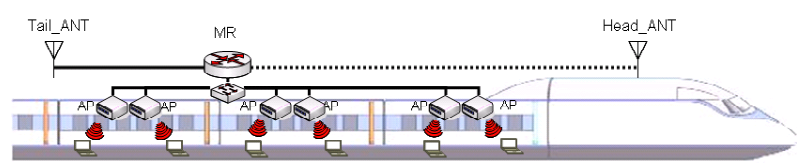

Fig. 1. Vehicle network structure of the proposed scheme 


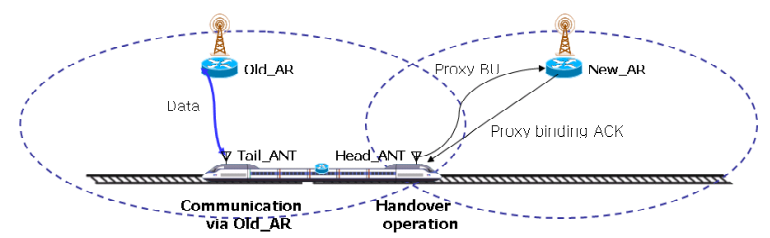

(a)

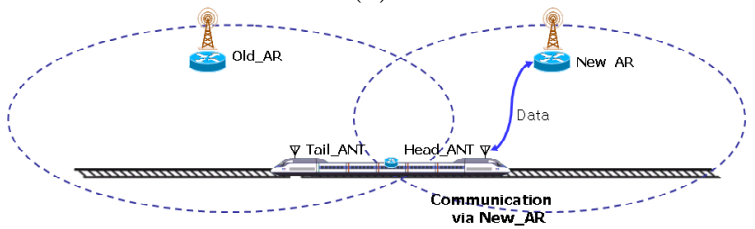

(b)

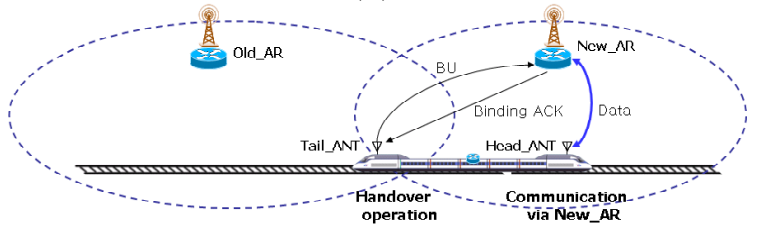

(c)

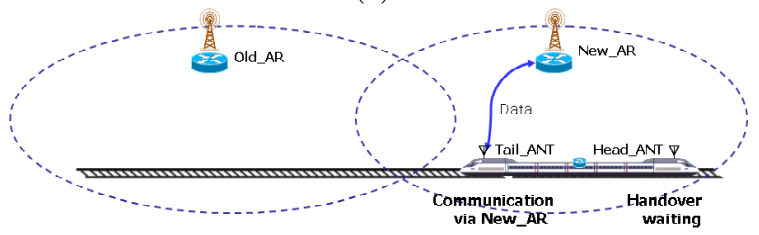

(d)

Fig. 2. Handover procedure of the proposed scheme (a)Phase 1, (b)Phase 2, (c)Phase 3 , and (d)Phase 4

sends a general BU message including the Tail_CoA and Tail_HoA through the New_AR. After receiving a Binding ACK message, the MR can send and receive packets through the Tail_ANT.

(4) Phase 4: When both antennas stay in the New_AR's coverage area, the MR communicated with the New_AR through the Tail_ANT, while the Head_ANT waits for an impending handover again.

In the proposed scheme, the proxy BU and the proxy BU ACK messages are newly introduced. The formats of these messages, however, are the same as those of the general BU and BU ACK messages in Mobile IPv6. The only difference between the proxy BU message and the general BU message is about the content of the messages. That is, the MR inserts the Tail_HoA into the Proxy BU message in place of Head_HoA. Table 1 shows the binding information maintained in the HA. 
Table 1. Binding information in the HA

\begin{tabular}{|c|c|c|}
\hline Phases & HoA & CoA \\
\hline Phase 1 & Tail_HoA & new Head_CoA \\
\hline Phase 2 & Tail_HoA & new Head_CoA \\
\hline Phase 3 & Tail_HoA & new Tail_CoA \\
\hline Phase 4 & Tail_HoA & new Tail_CoA \\
\hline
\end{tabular}

\section{Performance Evaluation}

Two critical performance issues are service disruption time and packet loss during handovers. For performance analysis, we use parameters as follows: total handover latency $\left(T_{H O}\right)$, movement detection delay $\left(T_{M D}\right)$, CoA configuration delay $\left(T_{C o A-C o n f}\right)$, delay for BU $\left(T_{B U}\right)$, router advertisement interval $(\tau)$, round-trip time between $\mathrm{MR}$ and $\mathrm{AR}\left(R T T_{M R-A R}\right)$, and round-trip time between $\mathrm{AR}$ and HA $\left(R T T_{A R-H A}\right)$. In this paper, we regard the service disruption time as the total handover latency, $T_{H O}$. The total handover latency the NEMO basic solution is given by:

$$
\begin{aligned}
T_{H O} & =T_{M D}+T_{C o A-\operatorname{conf}}+T_{B U} \\
& =2 \tau+2 R T T_{M R-A R}+R T T_{A R-H A}
\end{aligned}
$$

Since packet loss does not occur during the time when the CN traffic travels from the HA to an MR after the completion of the BU, the packet loss period $\left(T_{\text {loss }}\right)$ during a handover can be expressed as $T_{H O}-0.5 R T T_{M R-H A}$.

Packet loss ratio $\left(\rho_{\text {loss }}\right)$ is defined as the ratio of the number of lost packets during a handover to the total numbers of transmission packets in a cell. This can be also expressed as:

$$
\rho_{\text {loss }}=\frac{T_{\text {loss }}}{T_{\text {cell }}} \times 100
$$

where $T_{\text {cell }}$ is the time it takes an MR to pass through a cell.

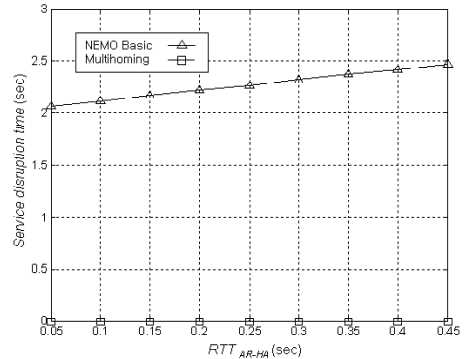

Fig. 3. Service disruption time

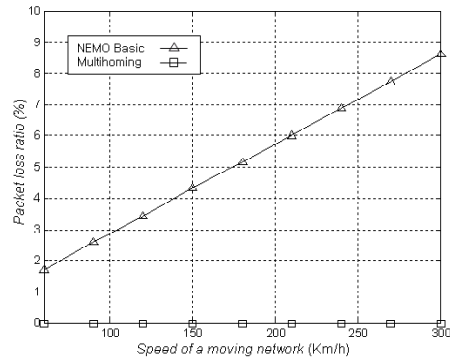

Fig. 4. Packet loss ratio 
However, in the proposed scheme, handovers of the Head_ANT and the Tail_ANT alternate with each other, thereby the total service disruption time and packet loss will be zero. Fig. 3 and 4 compare the service disruption time and packet loss ratio between the proposed scheme and the NEMO basic support, respectively. We assume that the router advertisement interval is 1 second, the radius of AR cell coverage is $1 \mathrm{~km}$, and $R T T_{M R-A R}$ is $10 \mathrm{msec} . R T T_{A R-H A}$ is assumed to be $100 \mathrm{msec}$ in Fig. 4. As shown, the service disruption time and packet loss ratio of the proposed scheme will be zero.

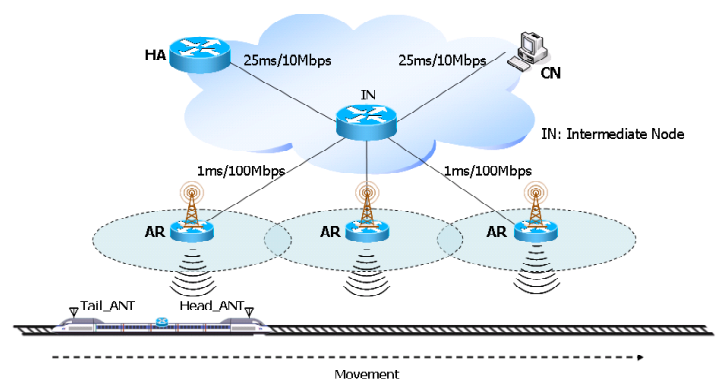

Fig. 5. Network model for simulation

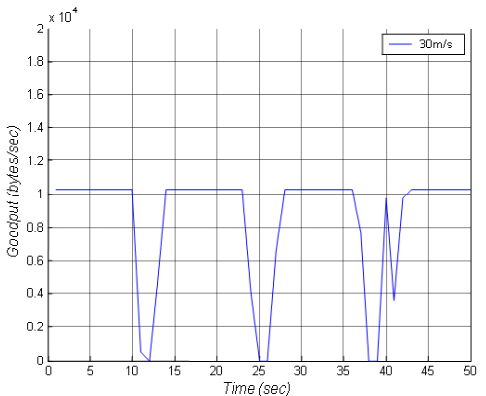

(a) NEMO basic (UDP)

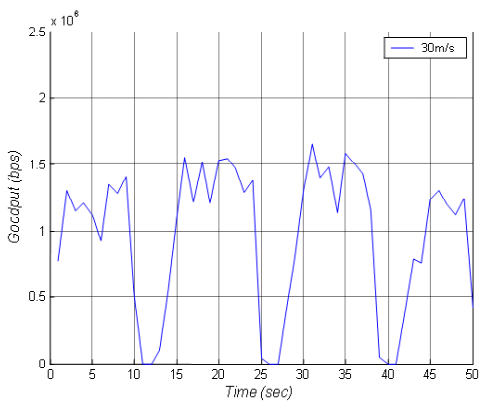

(c) NEMO basic (TCP)

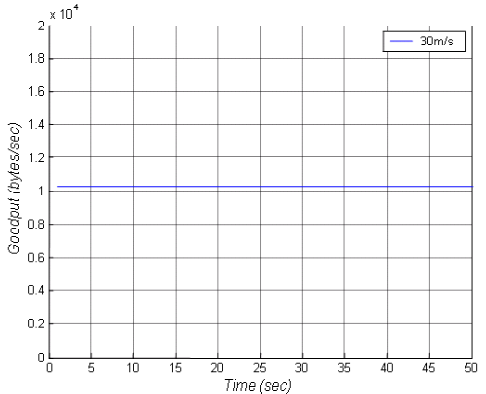

(b) Multihoming (UDP)

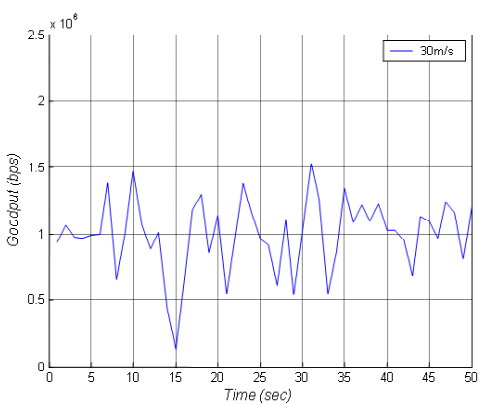

(d) Multihoming (TCP)

Fig. 6. Comparison of the UDP and TCP goodput behaviors at the speed of $30 \mathrm{~m} / \mathrm{sec}$ 
Fig. 5 shows the network model for simulation: Coverage radius of an AR is $250 \mathrm{~m}$, distance between ARs is $400 \mathrm{~m}$, router advertisement interval is $1 \mathrm{sec}$, IEEE $802.11 \mathrm{~b}$ as the wireless LAN, and distance between dual antennas is $200 \mathrm{~m}$. We have simulated for two traffic types: UDP and TCP. For UDP, the 512-byte packets were sent repeatedly at a constant rate of 20 packets per second from the $\mathrm{CN}$ to a mobile network node (MNN) residing in the train. For TCP, FTP traffic was generated with a full window. Fig. 6 compares the UDP and TCP goodput behaviors between the proposed scheme and the NEMO basic, respectively. From this figure, we note that the proposed scheme can provide a higher goodput in both cases of the UDP and the TCP, because the proposed scheme has no service disruption during handovers.

\section{Conclusion}

This paper proposed a seamless handover scheme using a multihomed MR with dual antennas for trains. Each of the dual antennas is located at each end of a mobile network for space diversity. One of the two egress interfaces of the MR can continuously receive packets through its antenna, while the other is undergoing a handover. Therefore, the proposed scheme can provide no service disruption or packet loss during handovers. However, the proposed scheme has some overhead in comparison with NEMO basic support. The overhead involves the cost to maintain dual MRs with additional signaling messages.

\section{Acknowledgment}

This work was supported in part by the KOSEF (contract no.: R01-2003-00010155-0) and the ITRC of the Ministry of Information and Communication (MIC), Korea.

\section{References}

1. V. Devarapalli et al., "Nemo basic support protocol," IETF RFC 3963, Jan. 2005.

2. E. K. Paik and Y. H. Choi, "Prediction-Based Fast Handoff for Mobile WLANs," in Proc. of ICT, vol. 1, pp. 748-753, Feb. 2003.

3. D. Johnson et al., "Mobility Support in IPv6," IETF RFC 3775, June. 2004.

4. C. Ng, J. Charbon, E. K. Paik, and T. Ernst, "Analysis of multihoming in network mobility support," InternetDraft, Feb. 2005.

5. N. Montavont, T. Ernst, and T. Noel, "Multihoming in nested mobile networking," SAINT2004Workshops, Jan. 2004. 